\title{
Temperature dependence of photoluminescence peaks of porous silicon structures
}

\author{
Róbert Brunner*, Emil Pinčík*, Michal Kučera ${ }^{* *}$, Ján

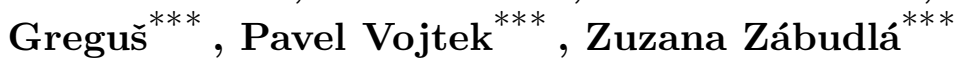

\begin{abstract}
Evaluation of photoluminescence spectra of porous silicon (PS) samples prepared by electrochemical etching is presented. The samples were measured at temperatures 30,70 and $150 \mathrm{~K}$. Peak parameters (energy, intensity and width) were calculated. The PL spectrum was approximated by a set of Gaussian peaks. Their parameters were fixed using fitting a procedure in which the optimal number of peeks included into the model was estimated using the residuum of the approximation. The weak thermal dependence of the spectra indicates the strong influence of active defects.
\end{abstract}

K e y w or d s: porous silicon, photoluminescence pectrum, Gaussian peaks, spectra evaluation

\section{Introduction}

In quantum confinement model proposed by Canham [1] the visible emission originates from electron-hole recombination between discrete energy levels inside the quantum wells formed by bulk silicon regions separated by nanoscale silicon particles (nanocrystallites). Luminescence of $\mathrm{Si}$ is often interpreted as a result of the presence of Si nanostructures of the scale of size 1-2 nm. Theoretical calculations of luminescence spectra for $\mathrm{Si}_{4}$ nanocluster (a planar rhombus) showing two major peaks at 530 $\mathrm{nm}$ and $650 \mathrm{~nm}(2.34$ and $1.91 \mathrm{eV})$ are reported.

Luminescence caused by Si nanoclusters has been observed in another Si-based material also, eg in silicon, silicon-rich oxide [2]. The photoluminescence (PL) of Si nanoclusters in silicon-rich dielectric materials was observed in the red to near-IR wavelength range. Two overlapping luminescence bands were found in this material: one of moderate intensity in the blue, and one of higher intensity in the red. Luminescence spectra contain broad maxima mainly located at approx. $820 \mathrm{~nm}(1.51 \mathrm{eV})$. The dielectric substrate can have some additional effects on the light emission process.

Deleure et al [3] have found that the red band of porous silicon (PS) $(1.4 \mathrm{eV} \quad 2.2 \mathrm{eV})$ could come from electron-hole recombination in crystallites with diameter between $2.5 \mathrm{~nm}$ and $4.5 \mathrm{~nm}$. Hernandez et al [4] have identified eight elementary photoluminescence bands for a-Si containing embedded nanoclusters of $\mathrm{Si}$ : 1.21-1.26 (I), 1.34-1.38 (II), 1.43-1.53 (III), 1.63-1.68 (IV), 1.77 (V), $1.87(\mathrm{VI}), 1.97$ (VII) and 2.06 (VIII) eV. The first four $\mathrm{PL}$ bands were explained as a radiative transition between quantum confined levels within Si quantum dots of a middle size $(3.19-3.95 \mathrm{~nm})$ embedded into the a-Si ma- trix. The last four PL bands are attributed to the surface state involved $\mathrm{Si}=\mathrm{O}$ bonds on the small size $\mathrm{Si}$ quantum dot surface $(0.55 \mathrm{~nm})$.

A model of size-dependent luminescence from localized states was developed by Estes and Moddel [5]. Highly confined amorphous structures exhibit a blue shift and an increase in quantum efficiency of the radiative emission. These effects are actually due to the statistics of spatial confinement in an amorphous semiconductor [6]. Simultaneously, the authors have explained the apparent contradiction between the evidence for localized transitions versus the evidence for size dependence, which implies delocalized transitions.

The PS includes silicon nanocrystallites (NCs) in the form of nanowires on the surface layer of monocrystalline silicon with different phases of crystalline c-Si and amorphous a-Si, covered with oxides $\left(\mathrm{SiO}_{y}\right)$ and hydrides $\left(\mathrm{SiH}_{x}\right)$. The nature of light-emitting centers PL is still not fully established and different models are offered for its explanation [7]. One of the earliest and the most widely used models is the quantum model of PL in which the luminescence is determined by the recombination of excitons in the NCs. Another model suggests that the luminescence related to hydride $\left(\mathrm{Si}-\mathrm{H}_{x}\right)$ bonds on the surface of nanocrystalline PS. There is also a model of the PL related to the presence of defect centers in oxides $\left(\mathrm{SiO}_{y}\right)$ at the interface of $\mathrm{NCs} \mathrm{PS} / \mathrm{SiO}_{y}$. The most widely accepted model for explaining the maximum intensity of $\mathrm{PL}$ at $\lambda_{\max }=640 \mathrm{~nm}$ associated with the defective levels of complexes hydrides and oxides on the surface of nanocrystallites (NCs), such as $\mathrm{SiH}_{x}$ or $\mathrm{SiO}_{y}(x, y=1-4)$. Maximum PL intensity at $\lambda_{\max }=440 \mathrm{~nm}$ related to radiative recombination of excitons in NCs PS [8].

* Institute of Physics, SAS, Dubravska cesta 9, 84511 Bratislava, Slovakia, robert.brunner@savba.sk, ** Institute of Electrical Engineering SAS, Dubravska cesta 9, 84104 Bratislava, Slovakia, *** Faculty of Mathematics, Physics, and Informatics of Comenius University, Mlynska dolina, F2, 84228 Bratislava, Slovakia 
In this paper, we present the results of photoluminescence experiments on PS. For the evaluation of PL spectra we have used a decomposition procedure based on fitting the measured spectra by a set of Gaussian peaks. The number of peaks in the spectrum was determined using the residuum of the fit.

\section{Experimental part}

An electrochemical method was used for preparation of samples. Silicon wafers of P-type (thickness: 600-650 $\mathrm{nm}$, resistance: 8-12 $\Omega \mathrm{cm}$ ) were cleaned in $\mathrm{HF}$ vapor during $30 \mathrm{~s}$, rinsed in distilled water, subsequently in methanol and dried in air. In the second step the sample was etched in a mixture of $\mathrm{HF}$ and methanol (mixing ratio 1:2), during $5 \mathrm{~min}$. A current of $50 \mathrm{~mA}$ was applied in the process. The repared sample was rinsed in distilled water and inserted into ethanol. Finally, it was dried in vacuum at ambient temperature.

PL measurements were performed in a liquid He cooled apparatus described in detail in [9]. The wavelength of the exciting light was $488 \mathrm{~nm}$. Measurements were performed at temperatures 30,70 and $150 \mathrm{~K}$.

The presence of a fine structure produced by the etching process is the dominant factor controlling the PL in porous silicon. Due to low temperatures applied during sample preparation we suppose that role of $\mathrm{SiO}_{x}$ complexes will be less important in this case and quantum confinement in nanocrystalline-like structures on the sample surface is the dominant mechanism controlling the PL effect.

Evaluation of PL spectra has been performed by fitting of individual peaks by a Gauss function [9]. This is well applicable for structures composed of relatively homogeneous domains (usually layers). Photon emission is an individual event, which is independent of other emission acts and is characterized by a specific spectral distribution of energy. Its independence allows to apply the central limit theorem leading directly to the Gaussian peak profile. If more PL processes take place in the sample (it contains different types of light emitting centers), the spectrum may be composed of a set of Gaussians.

Parameters of peaks were calculated via fitting of measured spectra by set of Gaussian peaks of proper parameters (energy, intensity and width FWHM) and constant bias representing noise. The root-mean-square (RMS) value was used as a merit function. The PL signal was pre-processed using loess transformation with a tricube weight function and smoothing parameter $\alpha=0.02$ to eliminate noise which could deteriorate the accuracy of the computed results reducing the stability of computation. The main advantage of loess is that it is a nonparametrical method based on a local regression model. Consequently, in comparison with classical approaches that use global parametric fitting functions (usually polynomial), loess substantially reduces the increase of data set correlation.
Real data are always perturbed and, in consequence, spectral peaks can be estimated only with limited precision. Even worse, this process may be ambiguous. Mathematically, this is the result of the fact that approximation functions represent a non-orthogonal base. Regardless of physical background, the precision of approximation could be increased if more peaks were included into the model. Physically, only a restricted reasonable number of peaks, corresponding to real processes taking place in the sample, should be used in this way. For determination of this optimal number we utilized the dependence of the approximation error on the number of peaks used in the computational model. The residuum of the approximation function was used as the estimate of this error. This dependence shows a distinct "knee" (see Fig. 4) allowing to fix the optimal number of simulated peaks representing the significant part of measured signal power. In a typical case, where the noise amplitude is sufficiently small, residua on the left side of the dependence represent mainly the error caused by omitting of peaks, while the right side represents the contribution of noise and rounding errors. Using too few peaks we will neglect the real peak, or peaks of PL spectrum. Usage of more peaks introduces small, non-important maxima in the model, with small amplitudes, or even artefacts. Additionally, it uselessly increases the number of degrees of freedom of the model, consequently increasing the computational instability and its sensitivity to input errors.

\section{Results and discussion}

Figures 1-3 show PL spectra decomposed into two Gaussian peaks. Measurements were performed for temperatures 30, 70 and $150 \mathrm{~K}$. Corresponding tables show corresponding parameters of peaks. Figure 4 shows the dependence of the residuum of the approximation process for different numbers of peaks for different temperatures. It clearly illustrates that the signal composed of two peaks is the optimal model.

The spectra show only a small shift of both the overall maximum and individual Gaussian peaks in dependence on temperature. This fact can be explained as the effect of the high concentration of defects caused by low-temperature chemical procedures used during sample preparation. No passivation steps were applied in this case. In our opinion, in this way the formation of siliconoxygen bounds was suppressed and the PL is controlled by mainly by quantum confinement effects. The presence of two Gaussian components (with corresponding two energies) only in PL spectra is partly in opposition to some of our former unpublished results in which a third energy was observed at $1.8 \mathrm{eV}$. This fact is interpreted as a consequence of the used technology and will be studied in more detail. 


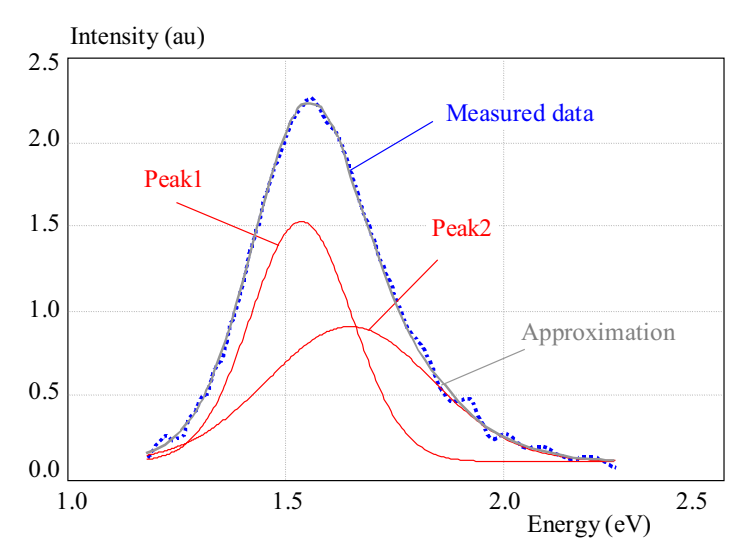

Fig. 1. EPOR30A, two peaks approximation at $30 \mathrm{~K}$

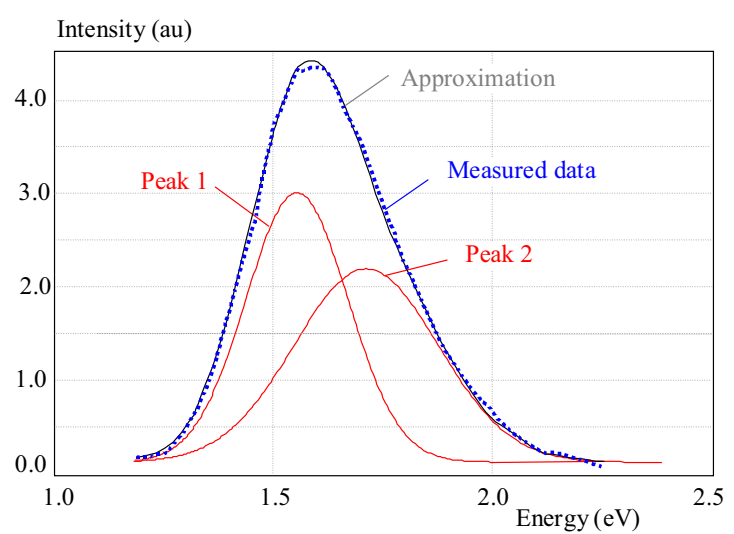

Fig. 3. EPOR150A, two peaks approximation at $150 \mathrm{~K}$

Table 1. Two peaks approximation: Temperature $30 \mathrm{~K}$

\begin{tabular}{lccc} 
No. & Energy & Intensity & FWHM \\
\hline 1 & 1.536 & $1.425 \mathrm{e}-6$ & 0.274 \\
2 & 1.647 & $8.013 \mathrm{e}-7$ & 0.444 \\
\hline
\end{tabular}

Table 2. Two peaks approximation: Temperature $70 \mathrm{~K}$

\begin{tabular}{lccc} 
No. & Energy & Intensity & FWHM \\
\hline 1 & 1.509 & $1.519 \mathrm{e}-6$ & 0.238 \\
2 & 1.652 & $1.836 \mathrm{e}-6$ & 0.348 \\
\hline
\end{tabular}

Table 3. Two peaks approximation: Temperature $150 \mathrm{~K}$

\begin{tabular}{lccc} 
No. & Energy & Intensity & FWHM \\
\hline 1 & 1.549 & $2.887 \mathrm{e}-6$ & 0.276 \\
2 & 1.712 & $2.067 \mathrm{e}-6$ & 0.397 \\
\hline
\end{tabular}

\section{Conclusion}

Porous silicon sample was investigated at temperatures 30,70 and $150 \mathrm{~K}$. We approximated each PL spectrum by a set of Gaussian peaks, where two Gaussians were used as an optimal model. Only weak thermal dependence of peak position was observed. This is explained by a high defect concentration. In comparison with our former measurements, an energy peak at $1.8 \mathrm{eV}$, related to the presence of $\mathrm{SiO}_{x}$ groups, was not observed.

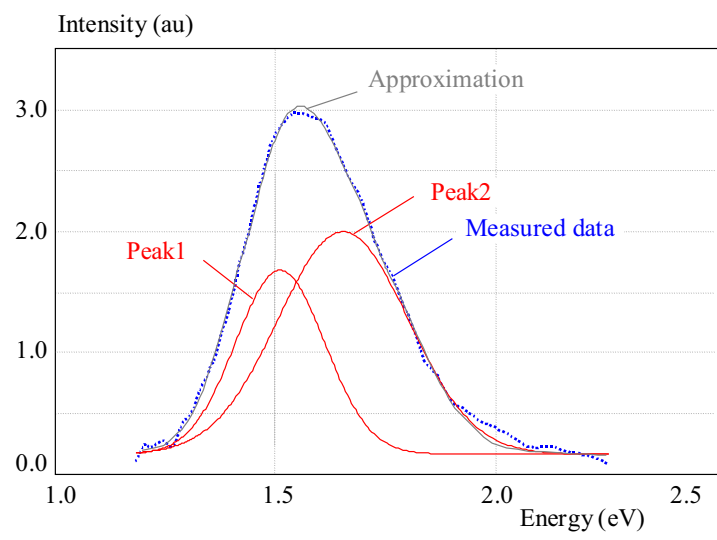

Fig. 2. EPOR70A, two peaks approximation at $70 \mathrm{~K}$

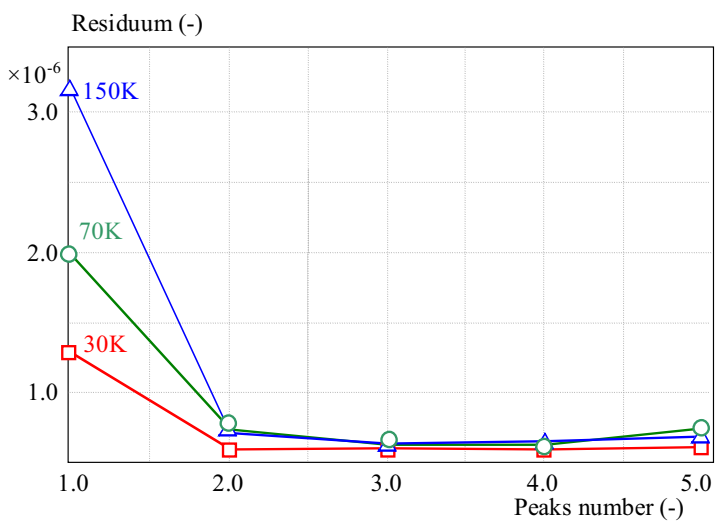

Fig. 4. Residuum calculated for 1 to 5 peaks used in the model

\section{REFERENCES}

[1] L. T. Canham, "Silicon quantum wire fabrication by electrochemical and chemical dissolution of wafers", Appl.Phys.Lett., 57 (1990) 1046-1048.

[2] M. L. Ciurea, "Quantum confinement nanocrystalline silicon", Optoelectron. Adv. Mater., 7 (5) (2005) 2341-2346.

[3] C. Delerue, E. Martin, J.-E. Lampin, G. Allan and M. Lannoo, "Luminescence of silicon crystallites", http://dx.doi.org/10.1051 /jp4:1993575.

[4] A. V. Hernandez, T. V. Torchynska, A. L. Q. Vazquez, Y. Matsumoto, L. Khomenkova and L. Shcherbina, Journal of Physics Conference Series 61, (2007) 1231-1235.

[5] M. J. Estes and G. Moddel, Phys. Rev. B 54 (20), (1996) 14 633-14 642.

[6] M. J. Estes and G. Moddel, Appl. Phys. Lett. 68 (13), 25 March 1996, 1814 - 1816 .

[7] A. S. Lenshin, V. M. Kashkarov, S. Y. Turishchev, M. Smirnov and E. Domashevskaya, "Influence of Natural Aging on the Photoluminescence of Porous Silicon", Technical Physics, vol. 57, no. 2, 2012, 305-307.

[8] N. E. Korsunskaya, T. R. Stara, L. Yu. Khomenkova, K. V. Svezhentsova, N. N. Melnichenko and F. F. Sizov, "The nature of emission of porous silicon produced by chemical etching", Semiconductors vol. 44, no. 1, 2010, pp. 82-86, doi:10.1134/S10637826 10010136.

[9] R. Brunner, E. Pinčík, H. Kobayashi, M. Kučera, M. Takahashi and J. Rusnák, "On photoluminescence properties of a-Si:H-based structures", Appl. Surf. Sci. 256(2010) 5596, http://dx.doi.org/10.1016/j.apsusc.2010.03.036.

Received 23 April 2017 